Background Improvements in care and treatment have led to more young adults with life-limiting conditions living beyond childhood, which means they must make the transition to adult services. A systematic review highlighted an acute lack of evidence on transition services for young people with palliative care needs.

Methods A realist evaluation approach using a mixed methods design with four phases of data collection was employed. Phase one involved the development and distribution of a questionnaire survey to health, social, educational and charitable organisations providing transition services to young adults with 104 individual responses from 29 organisations. Phase two involved interviews with eight young adults; phase three consisted of two focus groups with parents/carers and phase four involved interviews with 17 service providers. Data were thematically analysed seeking to identify organisational factors influencing the quality of care.

Results Eight interventions were identified associated with an effective transition. How interventions are considered to work were inferred and contextual factors were identified.

Conclusions Transition should be acknowledged as a key need for young adults with life-limiting conditions.An effective transition should be seen as a core responsibility for both children's and adult services. In addition to the transition interventions, there should be consideration for how the interventions work and the contextual factors that could influence whether the interventions are effective.

\section{USING A TRAFFIC LIGHT SYSTEM TO IDENTIFY PALLIATIVE CARE NEEDS IN ADULT NEUROMUSCULAR PATIENTS}

Derek Willis, R Vithlani, Tracey Willis. Chester University, Robert Jones Agnes Hunt Hospital, Severn Hospice

10.1136/bmjspcare-2018-ASPabstracts.206
The Department of Health has made a commitment to provide personalised and coordinated palliative and end of life care to those with life limiting conditions. Many neuromuscular diagnoses are or have the potential to be life limiting. Internationally there has been a lack of palliative service involvement or palliative approach to patients with such conditions.

Recognising that this was true of our neuromuscular service, we used quality improvement to develop a more proactive approach. As part of this project we developed a traffic light system based on 'The Spectrum of Children's Palliative Care Needs' used in paediatrics. This was adapted to be more relevant for neuromuscular patients. By reviewing their respiratory, cardiac, locomotor and gastrointestinal status and their recent hospital admissions patients are allocated a colour. Most importantly 'it would not surprise' the clinician if those who were 'red' died within twelve months or 'amber' patients died within a few years. 'Green' patients are currently stable and for 'blue' patients their condition is not expected to be life limiting. This tool would identify where patients were in their disease progression and highlight those who might benefit from a palliative approach, discussing advanced care planning or being seen in our newly established symptom control clinic.

All our patients with a palliative or potentially palliative diagnosis were allocated a traffic light colour and a system was developed to regularly review this. We used this to prioritise those patients with the most pressing need and consider how to best meet their needs. Figures for this will be available when the poster is published. It was not available as a full year of MDTs had not been run until the moment when late breaking was announced. 